\title{
El chino como lengua puente para la traducción al español de textos alemanes
}

\author{
Dong Xu He \& Ma . del Carmen Balbuena Torezano \\ Universidad de Córdoba \\ dongxuhe26@gmail.com \& mcbalbuena@uco.es \\ https://dx.doi.org.10.12795/futhark.2016.il I.04
}

Fecha de recepción: 15.10.2016

Fecha de aceptación: 20.11.2016

Resumen: En el presente trabajo se abordarán las dificultades de traducción de textos en los que hay temática agroalimentaria y turística, específicamente aquellos pertenecientes a la conocida fiesta de la cerveza alemana, el Oktoberfest. Se tratará el caso particular de la traducción alemán-español de este tipo de textos por parte de nativos cuya lengua materna es el chino. De este modo, se establecerá la utilidad de usar el chino como lengua "puente" para la correcta traducción del texto al español. Para ello, a partir de tres textos seleccionados, se comentarán las principales dificultades de traducción, así como la propuesta de traducción. Acompaña al análisis un glosario trilingüe alemán-español-chino, basado en la traducción a ambas lenguas, el chino y el español, de los textos originales en alemán.

Palabras clave: traducción especializada, terminología, chino, dificultades de traducción.

\section{Chinese as a Bridge to translate German Texts into Spanish}

Abstract: This paper addressed the difficulties of translation in terms of agro-food and tourism, especially for those agro-food in Oktoberfest which is the famous Germany beer festival. The particular case of the German-Spanish translation in such texts by speakers whose mother tongue are Chinese will be treated. Therefore, regarding Chinese as a "bridge", is also a good way to correct translation errors between German, Spanish and Chinese and could establish a close relationship between those three languages. This paper discussed the main difficulties of translation and translation proposal through three selected articles. Analysis accompanied a German-Spanish-Chinese trilingual glossary, which is based 
on the translation of three languages. The original articles are written in German and the target translation languages are Chinese and Spanish.

Key words: specialized translation, terminology, Chinese, translation difficulties.

Sumario: Introducción. I. La cerveza como elemento cultural: el Oktoberfest. I.I. El origen del Oktoberfest. I.2. La evolución del Oktoberfest. I.3. El Oktoberfest en la actualidad: las cifras de la edición de 2015. I.4. El Oktoberfest en otros paises. 2. El chino como lengua puente en la traducción de textos sobre el Oktoberfest. 2.I. La traducción de textos relacionados con el Oktoberfest. 2.2. Traducción y análisis de las dificultades de traducción de un texto tipo. 2.2.I. Dificultades de traducción: plano léxico-semántico. 2.2.2. Empleo de la lengua china para solucionar dificultades de traducción en el plano léxico-semántico.

\section{Introducción}

La traducción de textos agroalimentarios presenta una especial dificultad, pues se trata de una traducción especializada. Es necesario conocer una terminología específica para poder realizar con éxito la traducción del texto.

La dificultad para traductores cuya lengua nativa es el chino es aún mayor, si la combinación de lenguas de trabajo es alemán-español. En el presente trabajo abordaremos las dificultades de traducción de un nativo chino en el caso de textos alemanes relacionados con el Oktoberfest hacia el español.

Para ello partimos de la siguiente hipótesis: dado que la lengua materna del traductor es el chino, la traducción alemán-español se hace muy difícil. Por ello, es posible emplear el chino como lengua "puente" para la traducción de los textos, desde el alemán al español.

Tres son los objetivos fundamentales de este estudio:

I. Analizar la terminología propia de la cerveza y de la fiesta de la cerveza alemana (Oktoberfest).

2. Estudiar los procesos de traducción en los que el chino interviene como lengua "puente" para la traducción alemán-español.

3. Analizar las dificultades de traducción de los textos.

Para alcanzar los objetivos propuestos, el trabajo se estructura en las siguientes partes:

a) Una primera parte que analiza la producción de cerveza, el proceso de elaboración y los ingredientes. 
b) Una segunda parte en la que se establecen los tipos de cerveza alemana.

c) Una tercera parte en la que se analiza la producción y el consumo de cerveza en Europa, así como en España y Alemania.

d) Una cuarta parte en la que se aborda el Oktoberfest como elemento cultural conocido en el mundo.

e) La quinta y última parte es la más importante, pues lleva a cabo la traducción de tres textos alemanes relacionados con el Oktoberfest, en los que se emplea la lengua china como lengua "puente" para la traducción de los textos. También se analizan las dificultades de traducción, y se proponen glosarios trilingües alemán-español-chino para cada uno de los términos.

\section{La cerveza como elemento cultural: el Oktoberfest}

\section{I.I. El origen del Oktoberfest}

El origen del Oktoberfest se debe a la celebración del enlace del Príncipe Luis I de Baviera y la Princesa Teresa de Sajonia-Hildburghausen el 12 de octubre de 1810., el prometido invitó a todos los ciudadanos de Múnich. Los invitados fueron los hospedadores y los fabricantes de cerveza etc., que se volcaron en la celebración. El cierre de los festejos culminó con la carrera de caballos organizada por la alta burguesía de Múnich, en honor a la princesa. La pradera, en la que tuvo lugar la carrera de caballos, se denominó Theresienwiese', abreviado en Wiesn'. Tras el éxito de los festejos nupciales, el siguiente año los muniqueses decidieron seguir celebrando la carrera de caballos sin el patrocinio real. En adelante, se decidió celebrar la carrera de caballos cada año por la misma fecha. Es decir, la instauración del Oktoberfest como fiesta anual se debe a voluntad de los ciudadanos.

Sin embargo, la carrera de caballos, también se celebra por la asociación agrícola una vez cada cuatro años en la feria agrícola de Baviera. En sus orígenes se presentaban cosechas agrícolas y se organizaban varias actividades con el fin de

1Theresien-Wiese significa "Pradera de Teresa" en Alemán, se trata de un descampado de aproximadamente 46 hectáreas. En uno de sus lados se sitúa la colosal estatua de Bavaria, del escultor Ferdinand von Miller.

2Die Wiesen, que significa "la pradera", los habitantes de Múnich también llaman así al Oktoberfest. 
fomentar el trabajo del campo y mejorar la economía local. Se le llamó Zentrallandwirtschaftsfest ${ }^{3}$ o Bayerisches Zentral-Landwirtschaftsfest.

Antes de prohibirse comidas y cervezas, no había muchos entretenimientos; tampoco había cerveza en el Oktoberfest, hasta que la primera licencia la obtuvo Anton Gruber en 1818. A partir de ese año se añadieron carruseles, columpios y puesto de tiro de pinchón en la fiesta. Al año siguiente, el Consejo Municipal de la ciudad estipuló oficialmente la celebración anual.

\section{I.2. La evolución del Oktoberfest}

Luego, siguió celebrándose y año tras año se fueron añadiendo atracciones de feria, desfiles de trajes tradicionales, así como la reunión de propietarios de cervecerías.

A lo largo de los más de doscientos años de historia del Oktoberfest, se dejaron de realizar las actividades recreativas hasta en 24 ocasiones, debido a los períodos de guerra o a causa de una epidemia de cólera.

En el primer centenario del Oktoberfest el consumo de cerveza alrededor de 120,000 litros, rompió el récord mundial de aquellos tiempos. En 1950, surge la tradición de la consigna de apertura "O zapftis", instaurada por Thomas Wimmer, el primer alcalde que abrió el primer barril de cerveza al grito de la misma y tras la cual dan comienzo los festejos. En 2010, la celebración tuvo carácter histórico, se realizó una carrera de caballos en trajes de época y se sirvió una cerveza especial. Todo para que los visitantes tuvieran la sensación de haber retrocedido en el tiempo y de encontrarse en la atmósfera del siglo XIX.

\section{I.3 El Oktoberfest en la actualidad: las cifras de la edición de 2015}

Durante los dieciséis días de fiesta en el recinto de solo 42 hectáreas, casi siete millones de personas visitaron Múnich, consumieron alrededor de ocho millones de litros de cerveza, medio millón de raciones de pollo asado, con un total de 120000 visitantes bebiendo cerveza al mismo tiempo y produjeron 900 toneladas de basura.

\subsection{El Oktoberfest en otros países}

\section{-Argentina}

El Oktoberfest es conocido como la Fiesta Nacional de la Cerveza en Argentina, se celebra en el pueblo de Villa General Belgrano ${ }^{4}$, en el que dos

3La Fiesta Agrícola y Ganadera es una exposición agrícola en Múnich que se lleva a cabo cada cuatro años. 
alemanes, Jorge Kappuhn y Paul Heintze, importaron la costumbre de Alemania. Con el tiempo los habitantes de este pueblo fueron creciendo y adquirieron como propia dicha costumbre. Comenzaron a construir edificios diferentes a los locales, con los estilos típicos europeos, con maderas, jardines, tejados rojos.... También disponían de su propia gastronomía con sus platos típicos. Lo mismo ocurría con las costumbres, la música, los bailes, las fiestas, la artesanía y el idioma.

En 1960 se organizaron actividades con un viejo carro, las orquestas y los grupos de bailes en el centro de pueblo, en honor a las delegaciones representativas de los distintos países (grupos de Alemania, Dinamarca, Escocia, España, Isla Canarias, Yugoslavia, Suecia, Portugal, Brasil, Grecia, Italia, Armenia y Ucrania.), que vistieron sus ropas típicas.

A partir de 1996 se llevan a cabo desfiles con carruajes, bailes, grupos de músicas y trajes típicos. También, se organiza el concurso de la Reina Nacional de la Cerveza. Cada año una gran cantidad de turistas llegan al pueblo para celebrar la fiesta con ellos, el consumo de cerveza y la industria cervecera crecen notablemente.

-Chile

La fiesta de cerveza en Chile es pequeña comparada con la alemana. Su joven historia se remonta 35 años atrás, cuando en la década de los 80el Club Manquehue y el restaurante "Der Müncher de Malloco" se aliaron para llevar a cabola primera fiesta de cerveza. En el año 2005 la propietaria del restaurante "Der Münchner de Malloco" dispuso que la celebración se convirtiera en una fiesta abierta para toda la comunidad dónde se celebraran las costumbres tradicionales alemanas.

\section{-China}

A partir de 1992 se instaura en Hong-Kong la fiesta cervecera conocida como Marco Polo German Bierfest, en la que se pueden degustar comidas alemanas (Pretzel, salchichas, cervezas etc.). Desde hace diez años, el festival cuenta con propia banda musical: The Notenhobler. La fiesta transcurre desde el 16 de octubre al 7 de noviembre. En la ciudad de Qingdao también se celebra el Oktoberfest, que a su vez es el segundo más grande del mundo. Fundada en 1903 por inmigrantes alemanes, es también una de las marcas cerveceras en China. Se celebra en la segunda semana de agosto durante dieciséis días desde el año 1991, con espectáculos, desfiles, exhibición de cultura cervecera, exhibición de marcas, concurso de fotografía, concurso de Kung-fu etc. Como la más grande fiesta de

4 Villa General Belgrano es un pueblo pequeño con arquitectura bávara, con 6260 habitantes, una localidad situada en el departamento de Calamuchita, en la provincia de Córdoba, Argentina. 
cerveza en Asia, cada año una decena de países, regiones y cervecerías participan en esta celebración, acogiendo a 3,5 millones de visitantes.

\section{-España}

Se ha celebrado en las siguientes ciudades de España: Madrid, Zaragoza, Barcelona, Calpe, Calella, Calvá. Entre las distintas ciudades la duración y las fechas varían: En el año 2014, se celebró la fiesta del 24 al 27 de septiembre en Madrid, del 9 al 17 de octubre en Zaragoza, del2 al II de Octubre en Barcelona, del 7 al 18 de octubre en Calpe, del 3 al 24 de octubre en Calella.

Barcelona: El Oktoberfest Barcelona se celebra en la Plaza Universo de Fira Barcelona Montjiic, en la que se dispone una gran carpa principal con más $4,500 \mathrm{~m} 2$, donde por la tarde tiene lugar la ceremonia inaugural con la apertura del primer barril de cerveza. También, hay otro espacio especial con atracciones solo para niños y familias. Durante los festejos se puede disfrutar de varias cerveza alemana, música en vivo y comidas típicas alemanas (codillo asado, salchichas blancas, salchichas estilo núremberg, grillwurst etc.)

一Japón:

El Oktoberfest se celebra en diferentes lugares cada año (Yokohama, Odiaba, Nara, Machida, Fukuoda, etc.). Debido a su gran popularidad, se celebra dos veces, el Spring Oktoberfest a finales de abril y Oktoberfest tradicional en la ciudad de Odiaba. Dentro de la carpa, se puede disfrutar de música folclórica alemana, orquesta y cervezas bávaras (Weihenstephaner, Paulaner, Spaten, HackerPschorr...)

-Brasil

El Oktoberfest se celebra en la ciudad de Blumenau desde hace 30 años, gracias a la llegada los primeros colonos alemanes en siglo XIX que conservaron sus tradiciones. Es la fiesta de cerveza más grande y más conocida del continente americano, todos los años llegan miles de turistas a asistir a esta celebración. Aparte de ofertar las actividades típicas que se dan en otras ciudades que también celebran el Oktoberfest como son la música, la danza, la gastronomía y las actividades culturales tradicionales, también ofertan el Concurso Nacional de Tomadores de Chopp (cerveza) todas las noches a las 22 horas en Vila Germánica, la Elección de la Reina y el concurso deportivo de tiro "Rey del Tiro". Si las personas acuden con trajes típicos alemanes, pueden obtener entradas gratuitas a los pabellones de la Vila. En la fiesta no solo se pueden probar cervezas internacionales sino también cervezas locales de diversas cervecerías de la ciudad, así como comidas típicas locales. Hay bandas y artistas folclóricos actuando a diario por toda la ciudad. 
En síntesis, el Oktoberfest actual no es solo una fiesta de la cerveza que se limita a Alemania, sino que se ha convertido en un evento mundial. Las anteriormente citadas son las más conocidas pero hay otras que no hemos nombrado como son: el Oktoberfest en Estados Unidos, en Irlanda, en México, en El Salvador, Perú... Gracias a esta celebración se considera que la cultura cervecera de Alemania se ha extendido por todo el mundo con ayuda de los siguientes elementos claves:

\section{Gran impacto del Oktoberfest en Múnich}

2. Alemanes viviendo en el extranjero que promueven su cultura

3. La necesidad de mercado

4. La popularidad de la cerveza.

5. Colaboración entre gobiernos y organización.

Aunque el Oktoberfest se celebra en varios países y está rodeado por distintas culturas, siempre posee unas características comunes: el tema principal alrededor de las cervezas alemanas, incluyendo espectáculos, desfiles, música alemana, comida y atracciones; al mismo tiempo que se mezcla con los elementos locales. El concurso de la Reina Nacional se celebra tanto en Argentina como en Brasil. También, se celebra un concurso de Kung-fu en China. A pesar de que todos se llaman Oktoberfest, no es igual que otras fiestas oficiales que tienen una fecha fija en todo mundo; la duración de fiesta depende de sus propios organizadores, puede durar de una semana a dos semanas. La frecuencia de la celebración también se puede variar, por ejemplo: en Japón puede hacerse dos veces al año. Se propaga constantemente en todas partes y no dudo de que se extienda por más y más ciudades.

\section{El chino como lengua puente en la traducción de textos sobre el Oktoberfest}

Como se ha indicado al inicio de este trabajo, el objetivo fundamental de este estudio es el análisis de los textos turísticos en alemán incluyendo los textos traducidos, glosarios, términos y dificultades de traducción con el objetivo de identificar el importante papel que tiene el chino para el trasvase desde dos lenguas extranjeras, el alemán (TO) y el español (TM). El chino desempeña un papel imprescindible para los traductores chinos, pues no poseen ninguna de estas lenguas como lengua materna, actuando como lengua "puente" entre los dos idiomas. 
Como indican López Alonso y Sére $(2001)^{5}$, dentro de los procesos cognitivos, ocupa un papel fundamental en la intercompresión: I. El nivel lingüístico, 2. El nivel cognitivo, 3. El nivel social". Dado que la lengua materna es la lengua china, no se alinea directamente entre alemán y español, por eso, hay problemas de compresión en el nivel lingüístico sin usar el chino antes de dominar la segunda lengua perfectamente, por lo que no alcanza niveles superiores. La falta de dominio del español para el correcto trasvase de los contenidos del TO dificulta la labor de traducción. Por ello, el chino se considera como una herramienta en el proceso de traducción para la traducción alemán-español por parte de nativos cuya lengua materna es el chino, siendo destacables los siguientes aspectos:

I. Es posible aprovechar el chino para entender léxicos, términos, segmentos cortos, con el fin de alinear entre la segunda lengua extranjera (alemán) y la tercera lengua extranjera (español).

2. Gracias al efecto "puente" del chino, es posible generar un sentido coherente con el TO. Por consiguiente, la traducción se convierte en una traducción desde lengua materna (chino) a la lengua meta del encargo de traducción (español), pudiendo distinguir dos procesos traslativos: a) Traducción del alemán al chino y b) Traducción del chino al español.

3. Finalmente, es posible analizar la traducción desde el chino al español, pero hay que tener en cuenta del texto original, con el fin de revisar de nuevo la traducción si hay deficiencias tanto en el sentido como en el nivel de lengua.

No cabe duda de que, al tratarse de una traducción indirecta, con frecuencia pueden aparecer divergencias sutiles entre los significados, debido precisamente a esa doble transferencia que a veces conduce a un juego de idiomas. Por tanto, hemos de tener en cuenta no sólo las ventajas de emplear el chino como lengua puente, sino también las posibles desventajas. En este trabajo, sin embargo, pretendemos centrarnos en las ventajas de aprovechar la lengua materna (chino) con el fin de ayudar en la traducción.

\section{I. La traducción de textos relacionados con el Oktoberfest}

A continuación se propondrán varias traducciones relacionadas con textos turísticos o especializados sobre la cerveza alemana y la celebración del Oktoberfest. Para ello, vamos a proponer una tabla en la que explicaremos el texto original en alemán, y la traducción al español que realizo. Pero, sin embargo, para llegar a esta

${ }^{5}$ http://ic2012.u-grenoble3.fr/OpenConf/papers/50.pdf. [Fecha de consulta: 10.05.2016]. 
traducción, para el hablante de chino, es importante realizar primero la traducción alemán-chino y a partir de aquí, la traducción al español.

Por eso, además de la traducción al español, incluimos una tercera columna con la traducción al chino, que sirve como texto puente para realizar la traducción al español.

\subsection{Traducción y análisis de las dificultades de traducción de un texto tipo}

A continuación ofreceremos la traducción de un texto relacionado con el Oktoberfest, que lleva por título CSU hat eine besondere Idee für Bessoffene, y que presentamos en la tabla siguiente:

\begin{tabular}{|c|c|c|}
\hline Alemán & Chino & Espaĩol \\
\hline $\begin{array}{l}\text { Die CSU }{ }^{6} \text { hat eine besondere } \\
\text { Idee für Besoffene }\end{array}$ & $\begin{array}{l}\text { 基督教社会联盟提出一个新奇 } \\
\text { 的想法给醉汉。 }\end{array}$ & $\begin{array}{l}\text { EI CSU tiene una idea especial } \\
\text { para borrachos. }\end{array}$ \\
\hline $\begin{array}{l}\text { Die Stadt, in der ordentlich } \\
\text { und zumindest zur Wiesnzeit } \\
\text { außerordentlich getrunken } \\
\text { wird, könnte ein zentrales } \\
\text { Ausnüchterungszentrum } \\
\text { gebrauchen. }\end{array}$ & $\begin{array}{l}\text { 为了维持城市的正常秩序或至 } \\
\text { 少在啤酒节期间的安定, 提供 } \\
\text { 一个醒酒服务中心给酗酒者或 } \\
\text { 者醉酒者。 }\end{array}$ & $\begin{array}{l}\text { La ciudad, que está en ordeno } \\
\text { al menos, por el período de } \\
\text { Oktoberfest que se bebe } \\
\text { extraordinariamente, para ello } \\
\text { podria llevarse al centro de } \\
\text { desintoxicación etilica }\end{array}$ \\
\hline $\begin{array}{l}\text { Die Münchner Kliniken sollen } \\
\text { und werden schrumpfen, um } \\
\text { zu überleben. Über das } \\
\text { Konzept wird noch gestritten, } \\
\text { dennoch ist sehr } \\
\text { wahrscheinlich, dass } \\
\text { Krankenhaus-Gebäude in } \\
\text { Schwabing und Harlaching } \\
\text { frei werden. Die CSU fragt } \\
\text { sich nun: Was macht man } \\
\text { damit am sinnvollsten? }\end{array}$ & $\begin{array}{l}\text { 德国的医院为了维持运作将会 } \\
\text { 缩减。然而对于这个新的想法 } \\
\text { 认真正在积极的讨论中。但是 } \\
\text {, 很有可能的导致的是去斯瓦 } \\
\text { 宾格和哈莱辛的医院的人零垓 } \\
\text { 无几。因此, 基督教社会联盟 } \\
\text { 在考虑怎样做才能更有效。 }\end{array}$ & $\begin{array}{l}\text { Las clínicas en Múnich deberian } \\
\text { encogerse para sobrevivir. Del } \\
\text { concepto todavía se está } \\
\text { discurtiendo, pero es muy } \\
\text { probable que los edificios de } \\
\text { hospital en Schwabing y } \\
\text { Harlaching estén vacios. El CSU } \\
\text { plantea: "QQué es lo que hace } \\
\text { más sentido" }\end{array}$ \\
\hline
\end{tabular}

${ }^{6} \mathrm{CSU}$ es la abreviatura de Christilich Soziale Union (Deutschlands), Partido de la Unión Cristiano-Social de Alemania

Futhark II (2016) 


\begin{tabular}{|c|c|c|}
\hline $\begin{array}{l}\text { "Medizinisch nachnutzen", } \\
\text { schlägt der } \\
\text { gesundheitspolitische Sprecher } \\
\text { Hans Theiss vor. Und } \\
\text { präsentiert dafür ein } \\
\text { spezifisch Münchner Konzept: } \\
\text { Die Stadt, in der ordentlich } \\
\text { und zumindest zur Wiesnzeit } \\
\text { außerordentlich getrunken } \\
\text { wird, könnte ein zentrales } \\
\text { Ausnüchterungszentrum } \\
\text { gebrauchen. Einen Platz, an } \\
\text { dem besinnungslos Besoffene } \\
\text { "kontrolliert in einem } \\
\text { überwachten Umfeld } \\
\text { ausnüchtern" könnten. }\end{array}$ & $\begin{array}{l}\text { 卫生局发言人哈姆特斯提出采 } \\
\text { 用医疗方法对待醉酒者, 这一 } \\
\text { 特殊的概念对于慕尼黑人来说 } \\
\text { 是很特别的: ”为了维持城市 } \\
\text { 的正常秩序或至少在啤酒节期 } \\
\text { 间的安定, 提供一个醒酒服务 } \\
\text { 中心给酗酒者或者醉酒者。一 } \\
\text { 个可以给喝醉了无意识的人的 } \\
\text { 地方”. }\end{array}$ & $\begin{array}{l}\text { "El tratamiento médico", lo } \\
\text { propone el portavoz de la } \\
\text { Sanidad, Hans Tehsiss. Presenta } \\
\text { un concepto específico para } \\
\text { muniqueses: La ciudad, que } \\
\text { está en orden, al menos, por el } \\
\text { período de Oktoberfest que se } \\
\text { bebe extraordinariamente, para } \\
\text { ello podría llevarse al centro de } \\
\text { desintoxicación etílica. Un lugar } \\
\text { donde los borrachos } \\
\text { inconscientes podrían "ser } \\
\text { controlados en un entorno } \\
\text { monitorizado para } \\
\text { desintoxicarse". }\end{array}$ \\
\hline $\begin{array}{l}\text { Bisher werden diese in } \\
\text { Krankenhäuser eingeliefert, } \\
\text { was für das Personal und } \\
\text { die anderen Patienten in } \\
\text { den Notaufnahmen wenig } \\
\text { erfreulich bis gefährlich sein } \\
\text { könne, erklärt Theiss. Denn } \\
\text { nicht jeder schläft im } \\
\text { volltrunkenen Zustand } \\
\text { friedlich. Oder die } \\
\text { Rauschigen landeten bei der } \\
\text { Polizei, wo sie bei } \\
\text { Nichtbehandlung dauerhafte } \\
\text { Schäden erleiden oder } \\
\text { sterben könnten, erklärt } \\
\text { Theiss. }\end{array}$ & $\begin{array}{l}\text { 使这些喝醉了的人能被有效的 } \\
\text { 控制和使他们清醒。 } \\
\text { 就现在来说, 那些只有可能处 } \\
\text { 于危险的情况而被送往医院得 } \\
\text { 患者他们会感到一些不高兴。 } \\
\text { 由于不是所有的喝醉了的人的 } \\
\text { 反应是安静的睡觉, 还有一些 } \\
\text { 闹酒疯的人将会被送往警察局 } \\
\text {, 在那里由于没有接受到正确 } \\
\text { 处理引起持续性的伤害, 甚至 } \\
\text { 导致 }\end{array}$ & $\begin{array}{l}\text { Hasta el momento, ellos han } \\
\text { sido hospitalizados en el } \\
\text { hospital, lo que para personal } \\
\text { y los otros pacientes en la sala } \\
\text { de emergencias significa sentirse } \\
\text { algo más agradable/ algo } \\
\text { mejor, explica Thesis. Debido a } \\
\text { que no todo el mundo duerme } \\
\text { en el estado embriagado } \\
\text { totalmente con toda } \\
\text { tranquilidad. } 0 \text { bien los } \\
\text { ruidosos están arrestados en la } \\
\text { comisaría, en la que sin } \\
\text { tratamiento pudieran sufrir } \\
\text { daño duradero o incluso morir, } \\
\text { dice Theiss. }\end{array}$ \\
\hline
\end{tabular}




\begin{tabular}{|c|c|c|}
\hline $\begin{array}{l}\text { Die "zentrale } \\
\text { Ausnüchterungseinheit" } \\
\text { sollten Ärzte und die Polizei } \\
\text { gemeinsam betreiben. Falls } \\
\text { noch weitere } \\
\text { Krankenhausgebäude leer } \\
\text { stehen sollten: Für die } \\
\text { Wiesnzeit könnte die Stadt } \\
\text { bestimmt einen weiteren } \\
\text { Trakt anmieten. }\end{array}$ & $\begin{array}{l}\text { 醒酒服务中心将会配备医疗人 } \\
\text { 员和警察, 如果这样的话, 医 } \\
\text { 院会变得更空旷, 由于啤酒节 } \\
\text { 会有更多的医疗用地被征用。 }\end{array}$ & $\begin{array}{l}\text { El centro de desintoxicación en } \\
\text { el que deberían trabajar juntos } \\
\text { médicos y polícias. En caso de } \\
\text { que los edificios de hospital } \\
\text { estén más vacío: para la } \\
\text { Oktoberfest se pueden alquilar } \\
\text { los lugares al lado de edificios } \\
\text { de hospital }\end{array}$ \\
\hline
\end{tabular}

\subsection{Dificultades de traducción: plano léxico-semántico}

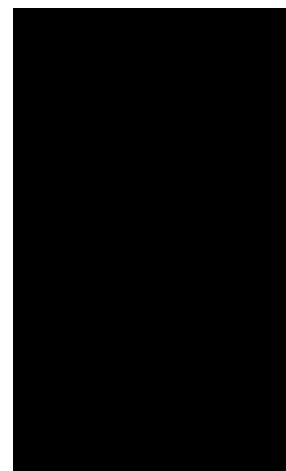

Dificultades de tipo

léxico
La expresión ein zentrales Ausnüchterungszentrum hace referencia a un centro dedicado a la desintoxicación etilica de personas en el Oktoberfest debido a que han bebido en exceso. Ausnüchterungszelle es la celda 0 el habitáculo para tratarlos, en cierto sentido es sinónimo de Ausnüchterungszentrum. Es muy fácil caer en la ambigüedad y confundirse con las expresiones "celda para desemborracharse", "centro para desemborracharse", "centro de desemborrachamiento", "celda de desemborrachamiento". En este caso, se va a traducir como "centro de desintoxicación etilica" porque Ausnüchterungszentrum, en virtud del significado de los dos elementos integrantes del Kompositum. Ausnüchterung (desintoxicación) + Zentrum (centro)

Wiesnzeit significa literalmente "el tiempo del Oktoberfest"; si bien pudiera pensarse que el término Wiesn está relacionado con los términos Wiese (prado) 0 Wiesen (prados), realmente es una abreviación de Theresienwiese, nombre dado a la explanada donde se celebra la fiesta en honor de Teresa de Sajonia-Hildburghausen.

Die CSU: die Christilich-Soziale Union in Bayern, se trata de la Unión Social-Cristiana de Baviera, que se fundó en 1945 y a partir de 1949 formó un grupo parlamentario con la Unión Democristiana de Alemania, solo realiza actividad en la región de Baviera.

Medizinisch nachnutzen. El término medizinisch es un adjetivo que se usa con sustantivo en el ámbito de gramática pero nachnutzen es un verbo. La composición puede traducirse como "tratamiento médico".

(...) einen weiteren Trakt. El significado del término Trakt es "ala"; en el contexto que aquí tratamos hace referencia a una zona determinada del hospital. 
2.2.2. Empleo de la lengua china para solucionar dificultades de traducción en el plano léxico-semántico.

Cuando por primera vez se ve un término desconocido como Ausnüchterungszentrum o Ausnüchterung y se busca el significado en el diccionario, aparecen tres acepciones en español: I) desintoxicación, 2) desemborrachamiento, 3) desembriaguez. La definición de "desintoxicación" es "acción y efecto de desintoxicar"; sin embargo, no existe una definición para el término "desemborrachamiento" en RAE, se trata de un derivado del verbo "desemborracharse" y es sinónimo de "desembriaguez". Como ninguno de los idiomas de los textos original y traducido es la lengua materna del traductor, lo que dificulta extraordinariamente su labor, pues trabaja con dos idiomas muy distintos al chino, resulta de gran utilidad emplear el chino para entender el término original, y, una vez encontrada la equivalencia en chino, es fácil comprender el concepto, y por tanto, trasvasarlo al español. La comprensión del concepto, gracias a la lengua china, nos lleva a traducirlo como "desintoxicación etílica".

Por su parte, el término Wiesnzeit hace referencia a los días de celebración del Oktoberfest. No hay una palabra coincidente con dicho vocablo, pues es una expresión genuinamente bávara. Sin embargo, en chino Wiesnzeit tiene la misma función que términos como Diestzeit (horario de trabajo) Essenszeit (hora de comer), Lebenszeit (duración de la vida), Friedenszeit (periodo de paz) o Hochzeit (boda), lo que puede ayudarnos a entender una expresión especial en alemán.

En el caso de Medizinish nachnutzen el problema está generado por la gramática, no se puede traducir directamente pues el segundo, nachnutzen, significa "reutilizar", por eso según el contexto hemos de deducir su sentido en chino, y tratar de buscar una forma correspondiente en español, que en este caso hemos optado por elegir "tratamiento médico".

\section{Conclusiones}

El análisis realizado en este trabajo permite las siguientes conclusiones:

I. La principal dificultad con la que se encuentra un traductor nativo de chino, que ha de realizar traducciones alemán-español es que trabaja con dos lenguas extranjeras: la primera lengua extranjera es el alemán (L2) y la segunda lengua extranjera, que también es la lengua meta, es el español (L3). Por ello, necesita la ayuda de su lengua materna (LI), que es el chino.

2. Debido a que el alemán y el español son idiomas flexivos, la formación de palabras es parecida en las dos lenguas ("Haupteingang" = Entrada 
principal; "Haupt" = principal, "Eingang" = entrada), y unos términos son más similares entre sí. En este caso, la función de la lengua china es establecer una relación entre dos lenguas extranjeras con el fin percibir las palabras a través del proceso cognitivo.

3. El traductor de textos alemán-español que no tiene como lengua materna el español encuentra una gran ayuda en su lengua materna, el chino, para comprender el sentido del texto original y luego traducirlo al español.

4. Sin embargo, en ocasiones, como traducimos textos que hacen referencia a una fiesta bávara, que sólo se da en Baviera, hay palabras que no tienen traducción; este sería el caso de términos como por ejemplo Loferl, donde el chino no sirve como lengua puente para ver su significado, lo que obligará al traductor a emplear otros recursos, como por ejemplo la búsqueda de imágenes, para entender el significado del término.

\section{Referencias bibliográficas}

BÉGUELIN-ARGIMÓN, V. El español como segunda o tercera lengua extranjera: algunos objetivos específicos. Disponible en red: http://crc.cervantes.com [fecha de consulta: 25.02 .2016 ].

CLYNE, M. (1997). Some of the Things Trilinguals Do. En: The International Journal of Bilingualism I, 2, 95-I I6.

Degache, C. \& Garbarino, J. (Ed.) (2012). Actes du colloque IC20/2. Intercompréhension: compétencesplurielles, corpus, intégration. Université Stendhal Grenoble 3 (France), 21-22-23 mayo de 2016.

VAN DE WALLE. (2015). Beer statistics 2015 edition, 3-36.

Wilke, V.; Villanueva de Debat, E.; Helale, G. (2010). Criterios para la elaboración de material didáctico para el desarrollo de la intercomprensión en lenguas germánicas. En: Segundas Jornadas Internacionales "Formación e Investigación en Lenguas Extranjeras y Traducción". Buenos Aires: Edición del IES en Lenguas Vivas, I5-20.

Recursos web

"Fiesta Nacional de La Cerveza- Oktoberfest Argentina" En: URL:https://www.elsitiodelavilla.com/oktoberfest/historia.html [fecha de consulta: 3I.03.20I6]

Heiner Effern, "Die CSU hat eine Besondere Idee für Besoffene" En URL:http://www.sueddeutsche.de/muenchen/staedtische-kliniken-die-csu- 
hat-eine-besondere-idee-fuer-besoffene-1.2937586 [fecha de consulta: 25.04.2016]

"La guía internacional del Oktoberfest" URL:http://www.oktoberfest.net/historia-del-oktoberfest/] [fecha de consulta: 02.04.2016]

"Oktoberfest: dónde saborear la tradición alemana"En URL:http://www.abc.es/viajar/restaurantes/201409/8/abci-oktoberfestespana-cerveza-201409161337.html [fecha de consulta: 24.04.2016]

"Oktoberfest en Blumenau: la mayor fiesta alemana de América" En:http://www.viajeabrasil.com/blumenau/oktoberfest-en-blumenau-alnordeste-de-santa-catarina.php [fecha de consulta: 23.04.20 I6]

"Oktoberfest Fiesta de la cerveza en Malloco, Chile" En:URL:http://www.fiestadelacerveza.cl/\#!blank-3/i8e4s [fecha de consulta: 02.04.2016]

"Sotragen Sie Ihre Loferl En URL:http://www.oktoberfest.de/de/article/Tradition/Tracht/So+tragen+Sie +lhre+Loferl+richtig/3295/ [fecha de consulta: 26.04.20l6]

“Tipos de cerveza en Alemania”. En: URL:http://jcsinprisa.blogspot.com.es/2008//2/tipos-de-cerveza-enalemania.html. [fecha de consulta: 09.03.2016]

"Wiese, Wiesen, Wies'sn oder Wiesn?". En URL: http://www.oktoberfest.de/de/article/Oktoberfest+2016/Service/Wiese, + Wiesen,+Wies'n+oder+Wiesn -2- /1294/[fecha de consulta: 08.05.2016] "Wiesn-Hits für Kids - Der Plan für die Familie". En URL:http://www.oktoberfest.de/de/article/Oktoberfest+2016/Familien/Wie sn-Hits+f\%C3\%BCr+Kids+-+Der+Plan+f\%C3\%BCr+die+Familie/ I234/ [fecha de consulta: 04.05.2016].

“青岛国际啤酒节” En: URL:http://www.qdbeer.cn/[fecha de consulta: 24.04.2016]

“慕尼黑啤酒节：酐畅淋漓的复古时尚”En URL: http://style.sina.com.cn/tas/wine/201 I-10-19/120885728.shtml_[fecha de consulta: 08.05.2016] 